\title{
Gender gap or program gap? Students' negotiations of study practice in a course in electromagnetism
}

\author{
Staffan Andersson ${ }^{*}$ and Anders Johansson ${ }^{\dagger}$ \\ Department of Physics and Astronomy, Uppsala University, Box 516, 75120 Uppsala, Sweden
}

(Received 30 January 2015; published 1 August 2016)

\begin{abstract}
[This paper is part of the Focused Collection on Gender in Physics.] This study of achievement differences, as reflected by course grades, on a third-semester electromagnetism course at a Swedish research university was motivated by instructor concerns about gender inequalities. Quantitative analysis showed a gender gap in course grades between female and male students for the period of fall 2007 to spring 2013. Dynamics behind this gap were explored through interpretative discourse analysis on interviews of 21 students who had recently passed the course. A recurring pattern was identified in the interviews. Students described studying electromagnetism as either studying to pass or studying to learn. Their choice of practice was influenced by the significance recognized in the course, which primarily was discussed in relation to program affiliation. Students stressed that perceived differences, in their study context, were larger between students affiliated with different programs than between male and female students on the same program. This was supported by quantitative analysis of course grades in relation to study programs, where the grade difference between female and male students on the same program in most cases were not statistically significant. The gender gap in grades for the whole course was related to different achievements on different programs. Programs further from the discipline of physics had lower mean grades and also enrolled a larger fraction of female students. Society-wide gender differences in interest and study choice are reflected in the grades on this single course. These results displace the achievement gap from the level of individuals to that of programs, and the gender gap from a difference in achievement to a difference in study choice. We discuss the implications of this shift of perspective in relation to gender differences for both research and teaching.
\end{abstract}

DOI: 10.1103/PhysRevPhysEducRes.12.020112

\section{INTRODUCTION}

Increasing diversity in science and engineering has been argued as a way to alleviate the shortfall of science and technology graduates that is experienced in many countries $[1,2]$. It is also seen as a matter of democracy and equality, as some groups of society progress differently or are not represented at all in certain fields of science at the moment. Calls for increased diversity have often focused specifically on gender [3] and the term gender gap is commonly used when describing, problematizing, and discussing quantitative contrasts between males and females [4].

In university physics, gender gaps have been explored in relation to factors such as participation, conceptual understanding, and achievement (course grades) [5]. However, there are still uncertainties, both regarding the reasons behind gender gaps and how to reduce or eliminate them.

\footnotetext{
*staffan.andersson@physics.uu.se

Also at Centre for Gender Research, Uppsala University, Box 527, 75120 Uppsala, Sweden.

Published by the American Physical Society under the terms of the Creative Commons Attribution 3.0 License. Further distribution of this work must maintain attribution to the author(s) and the published article's title, journal citation, and DOI.
}

This is, for instance, exemplified by conflicting results regarding the effects of interactive engagement techniques of teaching on closing the gap in conceptual understanding [5-7]. Recent studies argue that many different factors together contribute to the observed gaps [8]. For example, it has been suggested that cultural factors and norms play an important role [9]. Some attempts to use value affirmation interventions to reduce the negative effects of "identity" or "stereotype threat" have been shown to have at least a partly positive effect $[10,11]$. Other research discusses that measuring "equity" through decreasing gaps is not always straightforward, and that equity could be viewed in more ways [12]. Most research on gender gaps in physics education research (PER) has been conducted in the United States, but there are also some international studies [13].

Our study explores a gender gap in achievement in a course on electromagnetism at Uppsala University, Sweden. It originated from instructor concerns about perceived differences in course achievement between male and female students on the electromagnetism course. This study addresses the research question resulting from these concerns: What dynamics underlie the observed gender gap? Our answer to this question reveals general patterns for how these students interact with their course. This has 
implications concerning both educational practice and research on gender gaps.

\section{BACKGROUND}

\section{A. Context of the study}

This study is situated within the context of Scholarship of Teaching and Learning [14]. As part of strategic initiatives to scaffold the establishment of a community of scholarly educational practice at our university, stakeholders, such as instructors and students, are encouraged to raise questions that are explored in a scholarly fashion, often in collaboration with discipline-based education researchers.

The issue about possible differences in course achievement between male and female students raised by instructors on the electromagnetism course was initially explored quantitatively. There was no significant difference in pass rate for the course, where $74.4 \%$ of the female students and $74.7 \%$ of the male students passed the course for the period of fall 2007 to spring 2013. However, a significant difference was found for the course grades, reflecting the instructors' perception. Numeric grades for students passing the course are awarded on three levels: 3 , 4, or 5 depending on results on the final exam. A comparison between female and male students, using a two-tailed $t$-test, shows a significant gender gap in course grades ( $\Delta$ Grades $=0.29, p<0.001)$, as presented in Table I.

These initial results raised a specific concern about whether the course experience was in some way disadvantageous for female students. An investigation was initiated to explore underlying reasons to inform instructors in helping female students. This instructor-driven focus guided the first stage of the study, as initially only female students were recruited for interviews. This inductive, practice grounded approach, as discussed by Felten [14], provided insight into the question, but also indicated that more data was needed. Additional interviews were conducted to provide a broader sample of both male and female students, as results from the first stage strongly indicated that the observed grade difference concerned more factors than gender.

\section{B. The investigated course}

The electromagnetism course is a third-semester physics course taken by students on many programs within science and engineering at Uppsala University. The course is important as it forms an eligibility requirement for many

TABLE I. Average course grades for female and male students passing the electromagnetism course, and standard error of the mean.

\begin{tabular}{|c|c|c|c|c|c|c|c|}
\hline \multicolumn{3}{|c|}{ Females } & \multicolumn{3}{|c|}{ Males } & \multicolumn{2}{|c|}{ Difference } \\
\hline$N$ & Mean & SEM & $N$ & Mean & SEM & $\mathrm{F}-\mathrm{M}$ & $p$ value \\
\hline 348 & 3.35 & 0.03 & 791 & 3.64 & 0.03 & -0.29 & $<0.001$ \\
\hline
\end{tabular}

later courses in physics and other related fields, such as engineering and environmental studies. Students are formally required to pass courses in algebra, calculus, computational science, and introductory mechanics before enrolling in this course.

The course is taught through lectures, problem solving sessions, and laboratory exercises with some interactiveengagement elements, such as Peer Instruction [15] and collaborative problem solving $[16,17]$. The course material is the electromagnetism section of Sears and Zemansky's University Physics [18] with related internet material.

The course was given 18 times during the studied period from fall 2007 to spring 2013, 11 times with a female course leader and 7 times with a male course leader. However, in accordance with university policies for inclusive teaching, the course as a whole was taught by mixed gender teams from the physics department. All students met at least three different instructors. All instructors' educational backgrounds were in physics or engineering physics.

\section{Major programs on the course}

Swedish students aiming for a higher education degree, particularly in medicine, science, or technology, normally enroll in a study program. These study programs consist of a three- or five-year curriculum made up of suggested, and in many cases compulsory, courses. The content and profiles of these programs provide a multitude of choices for prospective students. The different profiles for the programs are often reflected in the social organization of students, for example, in program specific student associations, who can be said to maintain "program subcultures," with characteristic traditions, symbols, and other cultural expressions.

To exemplify, in the fall semester of 2014 there were 73 different five-year engineering programs offered at 12 different Swedish universities and university colleges, providing prospective engineering students with 123 distinct choices. These attract different populations of students due to different issues, many related to student views on who they are and who they want to become $[19,20]$. This difference is perhaps most clearly seen through the gendered patterns of choice. The enrollment database from the Swedish Council for Higher Education shows that some engineering programs, with profiles related to environment, medicine, and biotechnology, attract a majority $(\geq 60 \%)$ of female students, whereas some programs, with profiles related to computer science, electronics, and physics, attract a significant majority $(\geq 80 \%)$ of male students.

Students on the investigated course belong to 15 different programs. The majority of these students (1063 students, $93.3 \%$ of the population) were in one of the five study programs described below. Our study focuses on these students and their programs. Statistics for the programs are found in Table II. The qualifying merit point of 
TABLE II. The largest student groups passing the electromagnetism course, Fall 2007 to Spring 2013. The qualifying merit point is the minimum average grade from secondary education (high school), which was needed to be accepted into the program (average for 2008-2011). The merit point is calculated as an average of the students' best 17 grades from Swedish secondary education: Pass, Pass with distinction and Pass with special distinction, with numerical equivalents of 10,15 and 20 .

\begin{tabular}{lcccc}
\hline \hline Study program & $N$ & $f$ (females) $(\%)$ & $f$ (males) $(\%)$ & Qualifying merit point \\
\hline Energy Systems Engineering & 254 & 32.3 & 67.7 & 18.2 \\
Engineering Physics & 420 & 14.8 & 85.2 & 17.5 \\
Environmental Engineering & 198 & 69.7 & 30.3 & 18.2 \\
Materials Engineering & 118 & 22.9 & 77.1 & 13.1 \\
Physics Bachelor & 73 & 21.9 & 78.1 & 15.2 \\
\hline \hline
\end{tabular}

the programs, as presented in Table II, shows how hard it is to be accepted into a program and is seen as an indication of the capability and preparedness of students.

Engineering Physics is a five-year program with a focus on technical applications of physics. These students study two semesters worth of physics courses or more depending on choice of study direction, in fields such as applied physics or computational technology. The majority of the students enrolling in this program are male $(85.2 \%$ for the studied period).

Energy Systems Engineering is a five-year program with a focus on transformation and distribution of energy. These students study about two semesters worth of physics courses. The majority of the students enrolling in this program are male $(67.7 \%$ for the studied period).

Environmental Engineering is a five-year program with a focus on climate and environment. One semester of physics courses is part of the broad disciplinary base of this program. The majority of the students enrolling in this program are female $(69.7 \%$ for the studied period).

Materials Engineering is a five-year program with a focus on materials design. These students combine one and one-half semester of physics courses with courses in chemistry, technology, and mathematics. The majority of the students enrolling in this program are male $(77.1 \%$ for the studied period).

Physics Bachelor is a three-year program with a focus on physics, where students can choose between later specializations in physics, astronomy, and meteorology. These students take at least three semesters of physics courses. The majority of the students enrolling in this program are male $(78.1 \%$ for the studied period).

\section{METHODOLOGY}

\section{A. Theoretical framework}

To investigate the dynamics of the achievement gap, we conducted interviews with students about their practice and experiences of studying, in particular regarding the electromagnetism course. We adopt a discourse framework to make sense of these descriptions. This leads us to examine how students negotiate course practice in the enacted discourse of the interviews.

\section{Discourse and discursive identity}

Students' educational experiences have been found important for issues such as achievement [21,22] and retention [23,24]. Of particular interest to our research question is how students relate their experiences and actions to their conceptualizations of who they are, their discursive identity, especially in relation to gender. In our analysis, we view identity not as something fixed and connected to a single person. Rather, we interpret it as a socially negotiated position related to general cultural notions through language. This social constructionist view of identity is drawn from discourse theory, and to analyze the specific negotiations enacted in the language of interviews we use discourse analysis $[25,26]$. Specifically, we build on Gee's approach to discourse analysis where a central part is to identify different acts of construction performed through discourse, known as building tasks. The analysis of building tasks helps to understand the enacted discourse as their use reflects participants' understanding, interpretation, and construction of what is going on in a particular context. Gee identifies seven different building tasks (significance, practices, identities, relationships, politics, connections, and sign systems and knowledge) and shows how analysis can focus on these to investigate different types of questions. It is important to note that these views and modes of inquiry are mainly situated in a social constructivist paradigm of qualitative research, where the focus is on examining collectively constructed meanings, and not on, e.g., falsification of hypotheses [27].

\section{Perspectives on gender}

In our social constructionist, discursive view of identity, gender is not just a binary division between males and females, but a part of a social identity structured by and enacted through discourse. Still, we use gender to characterize the statistical "gender gap" which is the starting point of our study. One way of putting these things together is to consider the social construction of gender on several levels of analysis. Harding has formulated this as a call for studying gender on "individual, structural, and symbolic" levels, arguing that much research focuses on just one or 
two of these levels [[28], p. 52]. In the context of this research, we view individual gender as pertaining to students' individual negotiations of identity, structural gender as the broad distributions of women and men across different areas in society and in the university, and symbolic gender as the ideas and values about gender inherent in society and in certain subcultures, like parts of higher education. Additionally, gender is not the only social category that may come into play in education, but social background, ethnicity, and many other aspects often also interact in complex ways to shape an individual's identity or the specific inequalities she or he may be subjected to. An attempt at capturing this multiplicity is the concept of intersectionality, the analysis of the intersections between multiple categories and identities in social science $[29,30]$.

\section{B. Qualitative study}

\section{Qualitative data collection}

The qualitative data collection concerning students' experiences of the course was done through semistructured interviews [31], following a common interview protocol. The first part of the interviews dealt with general experiences of studying. The second part focused on achievement and experiences of the electromagnetism course. During the third part, students were asked to elaborate on possible reasons for differences in achievement on the course. Gender perspectives were raised explicitly in this last part.

As the motivation of the study, during the first stage in spring 2013, was a concern about female students' experiences of the course, respondents were found through a purposive sampling [32] among female students who had recently passed the course. Ten students participated in interviews during May 2013. Two students from Engineering Physics and two from Materials Engineering were interviewed individually. Three students from Energy Systems Engineering and three from Environmental and Water Engineering participated in focus group interviews. The switch to focus group interviews was done to encourage student interaction and enactment of program specific discourses. Preliminary analysis of data from the first stage indicated that gender played a less direct role than initially expected by course instructors.

This motivated a second round of data collection to gather a data set including male and female students from all major programs on the course. Two additional focus group interviews were arranged with students who had passed the course. The first focus group gathered two male students and one female student from Energy Systems Engineering and two male students and one female student from Environmental and Water Engineering. The second focus group gathered two female students and one male student from the Bachelor of Physics program, one male student from Materials Engineering and one male student from Engineering Physics. These interviews widened the qualitative data set to include male and female students from all major programs on the course.

All interviews were held in Swedish. Participants volunteered for the interviews and were informed that they could withdraw at any time and that the material would be treated confidentially before the recording started. The recording was done digitally and relevant parts of the interviews were transcribed. Analysis of the data was done in Swedish. The quotes used as illustration in this paper were subsequently translated to English. All quotes are labeled with gender and program affiliation of the informant, as these themes are relevant for the analysis.

\section{Interpretative discourse analysis}

The qualitative data were subjected to interpretative discourse analysis. Central for our analysis was the building task of practice [26], which concerns what practice the respondent constructs themselves as engaging in. Initial analysis identified all statements regarding practices related to studying - both electromagnetism and other courses. Those sentences were subjected to further analysis to identify other connected building tasks. Two other building tasks, identity and significance, were found in connection to statements regarding practice in the interviews.

We demonstrate our analytical approach through the following example from an interview, where the last part of the statement exemplifies the building task of practice-a practice of really learning things.

Well, I really have to know this, for the rest of my education and afterwards as well. Then I make really certain I learn it.

(female student, Engineering Physics)

The building task of significance concerns how the respondents construct things, such as the electromagnetism course, as being significant. The respondent describes in the middle part of the statement that, for her, the course has significance as being important for both the rest of the education and afterwards.

The building task of identity concerns whom the respondent wants or does not want to be seen as. It thereby relates to what different positions of identity the respondent perceives as possible in the context. The respondent constructs identity in the first part of the statement as she identifies herself as someone who needs to know the course content. This is also a disidentification in relation to some other students, who do not have to know the course content. In this interpretation, and when needed throughout the qualitative analysis, we relied on the broader context of the interviews and the original recordings for guidance when transcripts were ambiguous. In this particular example, both the emphasis put on the initial " $\mathrm{I}$ " in the statement and the context of how the respondent related to other students in nearby statements guided our interpretation. 
The instances of these three building tasks in the transcripts were coded, compared, and interrelated through an iterative process. This process yielded a recurring pattern that is presented in the results section.

\section{Quantitative analysis}

The quantitative analysis in this study used course grade data extracted from academic records for the 1139 students passing the course during the period of fall 2007 to spring 2013. This extraction was done by course administrators and resulted in anonymized tables with grade, gender, and program affiliation. All statistical analysis was done using Statistical Package for the Social Sciences, SPSS (version 21.0). Two-tailed $t$-tests were used for all comparisons, similar to, e.g., Ref. [5].

\section{RESULTS}

\section{A. Discourse regarding studying electromagnetism}

Interpretative discourse analysis of student statements revealed a recurring pattern that was present in all interviews. This pattern connects the three building tasks of practice, significance, and identity. The students describe a choice between two different practices: studying to pass and studying to learn.

We are always studying a bunch of courses, so one has to choose. Which do you work with properly? When is it enough to just ... if one just does them?

(male student, Energy Systems Engineering)

This choice of practice is described by the students as being motivated by the significance recognized for the course. Students discuss how this recognition of significance is affected by different aspects of their enacted social identity in relation to perceptions and experiences of the course. The two practices, studying to pass and studying to learn, in the student discourse about studying, primarily regarding the electromagnetism course, show strong similarities to what has been characterized as surface and deep approaches to learning [33]. The following sections present the practices of studying to pass and studying to learn with illustrative quotes from interviews.

\section{Studying to pass}

Students in all interviews discussed a study practice focused on finding out and meeting the minimum requirements to get a pass grade. We refer to this practice as studying to pass. Students who described adopting this practice on the electromagnetism course motivated this with the formal significance of the course as a requirement for future studies. All of the interviewed students needed at least a pass grade on this course to be eligible for registration on courses central for their programs in the following semesters. This was especially pronounced in the discourse of students from the Environmental Engineering program and the Energy Systems Engineering program. Students adopting this practice often described themselves as just "passing through" the electromagnetism course.

I don't feel that I need a higher grade than pass. Oh, but ... nice just to get rid of this.

(female student, Environmental Engineering) I believe attitude makes the big difference ... as we are satisfied with pass ... that is what's needed to continue onwards ...

(female student, Environmental Engineering)

The practice of studying to pass was described as a reasonable strategy. This was discussed in relation to program curricula with compulsory courses, some of which can be perceived as only having a formal significance as a prerequisite for other courses. To do more than what is required to pass the course is described by some students as a waste of time that could be spent on other more relevant courses.

When studying to become an engineer there are sometimes just a lot of required courses one has to pass without actually knowing, or even bothering, why. And if you're not interested in the content and don't know why you should really learn ... Yes, my ambition decreases ...

(female student, Engineering Physics)

Students often contrasted this practice, motivated by formal significance, to actually wanting to learn the course. Wanting to learn the course would require a motivation that they failed to find.

I felt I wouldn't have any use of this course later, other than as a prerequisite. So I didn't find it very interesting or exciting. And I didn't want to learn it.

(female student, Environmental Engineering)

\section{Studying to learn}

A practice of studying to learn was also described in all interviews, although in some cases just as something not adopted for the electromagnetism course. The aim of this practice is to really understand content and methods from the course to be able to use them in the future. Two different types of significance were described as motivation for adopting this practice-vocational and disciplinary significance.

I studied hard for that high grade as I have use for this knowledge later, when I've graduated.

(female student, Engineering Physics)

The vocational significance stresses the future usefulness and relevance of things learned from this course in a future vocation as a motivation for studying to learn. This was 
generally connected to a relatively clear view of a future career path, usually associated with electricity in some way.

I'll probably be working in an energy company after graduation ... I guess they would expect you to really know electromagnetism ... so I had to learn ... and that showed, I almost aced it ...

(female student, Energy Systems Engineering)

There were also examples in the interviews where students refer to vocational significance as something they failed to find in the electromagnetism course and that they therefore choose the practice of studying to pass. This was especially explicit in statements by students from Environmental Engineering.

Electromagnetism seemed narrower than I had expected. I couldn't see the use of it in the fields I plan to work in.

(female student, Environmental Engineering)

The disciplinary significance relates to the central role of electromagnetism within the discipline of physics. This significance was, on the other hand, mentioned as a motivation for studying to learn by students from the Engineering Physics and Bachelor Physics programs.

Electromagnetism is really core physics. As physics is supposed to be the brand of my program I made sure to learn it properly.

(male student, Engineering Physics)

Students from other programs related to it as a significance that only some of "those physics people" see. These students position themselves and their program in relation to the two programs they perceive as more "physicsy." There are also two examples in the interviews where meteorology students on the Bachelor Physics program distance themselves from the general physics direction.

Some go for the highest grades. They work so hard. It's primarily those who are straight into physics ... just for physics sake ...

(female student, Materials Engineering)

\section{B. Significance, identity, and gender}

Decisions about practice and recognition of significance were commonly described in the interviews in relation to aspects of identity. The students related this ongoing negotiation of practice to who they perceive themselves to be, but generally in rather vague terms.

\section{Self and significance}

Self-identification in connection to the course, as expressed in student statements, relates to three interconnected themes: program affiliation, future career plans and relation to the discipline of physics. Students from the same program often relate in similar ways, they can be said to draw from the same "discursive repertoire" formed in the program's culture.

Students' self-identification is regularly played out in comparison to other students who they perceive as unlike themselves. This is particularly pronounced when disassociating themselves from physics, in contrast to "other students who care about physics."

I am not like those who ... like ... love Maxwell's equations just because they are there. What good are they in real life? That's what I would like to know.

(female student, Energy Systems Engineering)

I am after all not someone who is as interested in ... in just these courses ... like some others ... this physics you just have to do.

(male student, Environmental Engineering)

Here, identity is constructed in relation to significance and practice. A constructed identity as someone who is not interested in "these courses" implies that the course is seen as lacking any personal significance and a practice of studying to pass is enacted. However, not only does the significance attributed to a course vary from person to person but the negotiation is also a dynamic process evolving over time.

Suddenly ... after half the course ... there was this example from my field of interest and I realized ... Ooops! This might actually be relevant for me ... for my future life ... and not just for physics. I changed my plans for the course entirely.

(female student, Environmental Engineering)

\section{Discussing the role of gender}

Although a gender perspective was central for initiating this study, students never raise the subject of gender spontaneously in the interviews. Rather, they talk more broadly about who they are, often in accordance with a program discourse regarding future careers and relationship to physics.

It is only when gender is explicitly brought up in the interviews that the respondents start to talk about it. However, they do not describe it as an important aspect in relation to the recognition of course significance. Rather, they problematize the idea of gender (or rather biological sex) as the primary categorization of individuals, at least on this level of the educational system.

I think that it is hard to just separate between male and female students. I don't understand why this should be the difference to start with. It's like a bigger difference between people with different directions of study ...

(female student, Engineering Physics) 
TABLE III. Mean course grades on the electromagnetism course for the five largest study programs.

\begin{tabular}{lcc}
\hline \hline Study program & Mean grade & SEM \\
\hline Environmental Engineering & 3.24 & 0.04 \\
Energy Systems Engineering & 3.44 & 0.04 \\
Materials Engineering & 3.49 & 0.07 \\
Physics Bachelor & 3.73 & 0.10 \\
Engineering Physics & 3.78 & 0.04 \\
\hline \hline
\end{tabular}

Some of the students express that although they are aware of the construction of gender as two dichotomous categories, it is not something they recognize as important in their current study context. They claim that belonging to different programs can create more opposing categories than being male or female in the same program.

There are opposites among the men as well ... just as with us women. Some want to understand it all and some are just happy to manage. If anything, the people within the same program are more alike ... but still not the same ...

(female student, Energy Systems Engineering) A: If there are differences ... that happens before you come here ...

B: I really don't think gender is so important at this stage. What would be the basis for that?

A: It's more like ... it's just on our program that we think like this.

(female students A and B, Bachelor of Physics)

There are references in this later part of the interviews to gender differences in interest and study choice on a broader scale. This is especially pronounced when students discuss how programs are marketed in different ways and how that, in combination with program content, could make programs more "masculine" or "feminine" and thereby more attractive to different parts of the youth population. This discussion is related to broad cultural factors, stereotypes and expectations.
When you are younger ... people might expect you to know more about electricity as a boy. That could influence how your interest forms ... and how you choose. But now I have the impression that most people on our program have similar interests ... both men and women. That's why we're here.

(male student, Materials Engineering) B: Maybe it is so that women choose more to study towards a purpose ... and not just to learn this physics ... but not all women ...

A: It's not that simple. I like astronomy ... and disliked electromagnetism ... but I don't think that's just because of my chromosomes. There is so much more ...

(female students A and B, Bachelor of Physics)

\section{Quantitative analysis}

Quantitative comparison between female and male grades for the whole student population showed a significant difference. However, during the interviews students repeatedly claimed that the difference between programs was larger than that between women and men, at least in the course context. This motivated a quantitative analysis in relation to programs.

The mean grades for all students of the five major programs are presented in Table III. There was no statistically significant difference in grade distribution between students on Energy Systems Engineering and Materials Engineering or between students on Physics Bachelor and Engineering Physics. However, all grade differences between the three groups presented in Table III are statistically significant $(p<0.001)$. Environmental Engineering, distanced from the discipline of physics and with a majority of female students, has the lowest mean grade. It is significant that the two programs with the highest qualifying merit points (Environmental Engineering and Energy Systems Engineering, see Table II), where students are expected to be best prepared, are those with the lowest mean grades for the electromagnetism course. On the other hand, in the interviews, the practice of studying to pass was especially pronounced among students from these programs. Physics Bachelor and Engineering Physics, most closely associated with the discipline of physics, have the highest mean grade.

TABLE IV. Comparison of course grades for female and male students passing the electromagnetism course on the five largest study programs.

\begin{tabular}{|c|c|c|c|c|c|c|c|c|}
\hline \multirow[b]{2}{*}{ Study program } & \multicolumn{3}{|c|}{ Females } & \multicolumn{3}{|c|}{ Males } & \multicolumn{2}{|c|}{ Difference } \\
\hline & $N$ & Mean & SEM & $N$ & Mean & SEM & $\mathrm{F}-\mathrm{M}$ & $p$ value \\
\hline Environmental Engineering & 138 & 3.23 & 0.05 & 60 & 3.25 & 0.06 & -0.02 & 0.82 \\
\hline Energy systems Engineering & 82 & 3.44 & 0.07 & 172 & 3.45 & 0.05 & -0.01 & 0.93 \\
\hline Materials Engineering & 27 & 3.44 & 0.13 & 91 & 3.51 & 0.08 & -0.07 & 0.71 \\
\hline Physics Bachelor & 16 & 3.63 & 0.20 & 57 & 3.75 & 0.11 & -0.13 & 0.58 \\
\hline Engineering Physics & 62 & 3.48 & 0.09 & 358 & 3.83 & 0.04 & -0.35 & 0.01 \\
\hline
\end{tabular}




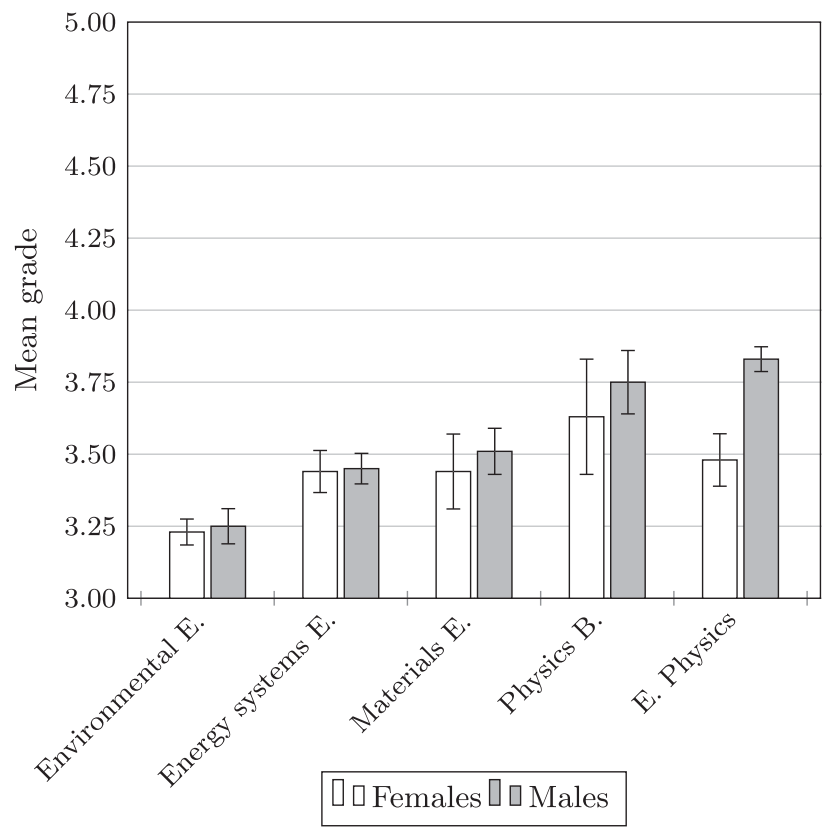

FIG. 1. Mean grades for female, male and all students on the five major programs on the course. Error bars indicate standard error of the mean.

The different grade distributions for the programs in combination with the different fractions of male and female students on each program could explain most of the accumulated gender gap in grades for the whole course population. Further comparisons were done to explore gender related differences within the five major programs. The results are shown in Table IV and illustrated in Fig. 1. Comparison of mean grades between female and male students within the same program shows a statistically significant gender gap for only one of the five programs: engineering physics. For the other programs, in particular the three "nonphysics" engineering programs, we find that female and male students exhibit similar results. These results give credibility to the qualitative findings regarding students' negotiations about their practice on an individual level, where recognition of significance and resulting study practice can be seen as more strongly influenced by program affiliation than by gender.

\section{DISCUSSION AND IMPLICATIONS}

The initial aim of our study, as formulated by course instructors, was to explore the apparent gender gap to inform gender-inclusive action on a student level. However, our results reveal broad dynamics involving issues of significance, culture, and identification. Although this study focuses on electromagnetism we believe these discussions have bearing on many parts of physics education. In this section we discuss our findings in relation to other research, what they mean for continued research on gender gaps, and the possibilities of more inclusive teaching.

\section{A. Discussion of results}

\section{Gender gap or program gap?}

In our analysis of the discourse in the interviews, we see students' negotiating the discursive practices of studying to pass and studying to learn, which are very similar to the deep and surface approaches to learning [33]. The process of choosing between these approaches has been discussed, for example by Ramsden [34]. Ramsden argues that "Approaches to learning are not something a student has; they represent what a learning task or set of tasks is for the learner" [[34] p. 45]. The interpretation of a task influences which approach, or practice, the students choose to adopt.

An interesting point in the discourse of our students is that this process of interpretation and choice, in relation to the electromagnetism course, is more strongly associated to their program affiliation than their gender. This necessitated a perspective shift from gender on an individual level, as framed in the initial instructor concerns, to broader cultural dynamics. The observed gender gap, as presented in Table I, is not simply due to male and female students performing differently on the course. Instead, we found that the grades of male and female students on most programs are similar. Interviewed students from the same program also relate to the course in similar ways in their discourse.

Our results indicate that the role played by gender in this situation does not lie on the level of the individual, but rather on the level of culture and discourse in a wider sense. We do not have similar male and female students performing differently. Rather, male and female students, as is evident from the gender distributions of the programs, (on average) choose study directions differently. This results in the overall gender gap on the course grades.

\section{Negotiating the culture of physics}

If the discursive negotiations of our interviewees are taken to represent students' negotiations of course practices on a wider scale, we suggest that these interactions could be understood as different degrees of "cultural clashes" between the subcultures of the programs and that of the course. Aikenhead discusses clashes between students from different cultural backgrounds and the culture of school science and argues that these clashes can explain the difficulties many students have when encountering school science [35]. Our study concerns students with different backgrounds and interests encountering university physics.

The electromagnetism course is taught by physicists at the physics department with an educational background in physics, and can be argued to reflect a "physics subculture" and physicist values. Students aiming for a career close to the physics discipline, especially physics bachelor or engineering physics students, often seem to find disciplinary significance in the course. This may be expected as 
these students probably participate more in the "culture of physics."

On the other hand, students not identifying with "physics for physics sake," or looking for vocational significance, might have a hard time finding anything but a formal significance for the course and thus enact a practice of studying to pass. There is a clear similarity between this behavior and that exhibited by students needing to achieve science credentials just to be eligible for post-secondary studies. Aikenhead describes how this places students in a situation of "having to play school games to make it appear as if significant learning has occurred even though it has not" [36] (p. 28).

As is clear in the interviews, students' negotiations of significance relate to their ideas of belonging to certain groups of people, in our case primarily to the study program. In the Swedish context, where programs are sometimes narrow and specialized, students from programs distanced from the discipline of physics might experience similar cultural clashes to those Aikenhead describes when encountering courses reflecting a strong physics culture.

Considering the identity negotiations of the students in our material in relation to previous research on study choice, we see many connections. Although the differences among programs are more important than gender with regard to results, the programs themselves have unequal gender distributions, reflecting wider patterns of study choice $[3,37]$. In research about school students' attitudes toward science, large surveys in many countries show how girls generally care more about environmental issues and boys more about spectacular phenomena and technology [38,39]. These patterns reflect major cultural ideas, where men and women are expected to be interested in different things and work in different areas. In this respect, physics is often described as masculine in several ways, and this has partly been taken as an explanation for the reluctance of women to choose physics [40-42]

When students make a decision about what to study, the gendered expectations in the dominant discourse come into play in a complex way with their individual identity negotiations. The symbolic level affects the individual, and this is reflected clearly on a structural level where we see a statistical gender imbalance in study choice as well as grades. Like us, recent studies on students' attitudes and study choice often employ a social constructionist, identity perspective to gain an understanding in the dynamics behind these processes [19,20,24,43-45].

\section{Remaining questions}

This instructor-initiated study provides inspiration for further studies on cultural aspects of university physics. Program affiliation affected how students related to the electromagnetism course. This negotiation of study practice would likely be different for other courses, especially regarding the recognition of significance. Comparative studies between programs and courses could explore this further.

Although the differences between programs can explain a major part of the achievement gap in the course, there are also other dynamics that are not explained through our study. There is a statistically significant gender gap in course grades on the Engineering Physics program. A similar tendency, although not statistically significant, is also seen for the Bachelor of Physics program. This difference occurs on the programs that are most closely associated with the discipline of physics. One possible contribution to this effect could be that these broader programs recruit a more heterogeneous population of students with varying ideas about physics and future careers [46]. Students from the Bachelor of Physics program and the Engineering Physics program often talk about the different directions of their program in the same way as other students speak about different programs. This diversity could perhaps affect the recognition of significance and choice of practice. There is also the possibility that female students on these programs with a more traditional physics discourse where women are a small minority could be affected by stereotype threat dynamics $[10,11,47]$. Further studies on these particular programs would be needed to understand this issue.

\section{B. Implications}

The importance and relevance of biological sex (or gender as specified in records) as a category for investigation, understanding issues, and informing action can differ on different levels, as illustrated by our study. This has important implications for both research and teaching.

\section{Implications for research}

The presence of a gender gap, as identified through a quantitative investigation, can be a clear indication of structural differences within a heterogeneous population. However, such a difference between male and female students in itself does not explain anything. Further studies are needed to elucidate the underlying dynamics. Qualitative methods can contribute nuanced descriptions and explanations, as well as provide new ways to view and interpret quantitative data. Our study exemplifies how such a mixed-methods approach can contribute to a broader understanding. Particularly, it points to the possible relevance of studying students' negotiations of culture and identity, something that could be a starting point for further studies.

Comparisons between male and female individuals, as two dichotomous categories, can certainly be useful in some contexts, perhaps primarily on a structural level concerning large populations. For our study, a shift from an individual level towards a more structural level was needed to better understand the dynamics behind the gender gap. This exemplifies the value of studying gender 
issues in science on several levels and as a part of an ongoing discursive identity negotiation working on an individual level in relation to culture-wide discourses. This deeper and broader understanding of gender has been argued as largely missing from research in science education $[48,49]$. Furthermore, this study shows the value of considering different categories in interaction with gender. Applying an intersectional perspective, we might be able to avoid glossing over the diversity within groups like male and female to get a more complete analysis.

\section{Implications for teaching practice}

Physics courses are an integral part of many different programs and cater to a diverse population of students. This becomes even more pronounced as the diversity of educational programs increases. In our studied context, there are numerous profiled programs recruiting select parts of the youth population. Marketing presentations of these programs exhibit a multitude of examples of doing physics in different contexts and allow many possibilities for identification. However, once on a program, students are often expected to take the same physics courses, especially during the early years of their education. This could entail doing physics in a more narrow physics culture, which they can have problems identifying with.

Discussions on physics teaching often stress the importance of recognizing and adapting to the diversity of physics students, but primarily in relation to cognitive aspects (cf. Ref. [50]). However, our study stresses the importance of not forgetting the cultural and social aspects. Many students in our studied context, primarily from programs at a distance from the disciplinary and vocational field of physics, failed to recognize anything but a formal significance for the electromagnetism course. This led them to a practice of studying to pass resulting in lower grades, and getting less out of the course than they could have. This, in turn, resulted in a gender gap of grades on a course level as these programs to a larger extent recruit female students.

Our results emphasize the importance of providing courses that are seen as significant for all students, to encourage both learning and participation. There are examples in the interviews of how just a single significant incident can make students change their practice. We suggest that examples, problem contexts, guest lectures and other parts of the course should be chosen from different areas, where physics is being practiced and applied. Our findings have motivated a reform of the studied course, where instructors now include problems and examples from various contexts, and guest lecturers from different vocational areas participate. Students, especially from fields further from physics, have been very positive to these changes in the course evaluations.

\section{ACKNOWLEDGMENTS}

The authors would like to thank instructors and students of the electromagnetism course. We thank editors, reviewers, and colleagues at the Division of Physics Education Research, the Centre for Gender Research, and the Centre for Discipline-Based Education Research in Mathematics, Engineering, Science, and Technology, Uppsala University for their many helpful comments and suggestions on this work. We are especially grateful to those of our colleagues who have read and commented the manuscript at different stages.
[1] European Commission, Europe Needs More Scientists: Report by the High Level Group on Increasing Human Resources for Science and Technology in Europe (Office for Official Publications of the European Communities, Luxembourg, 2004).

[2] A. Macdonald, "Not for People Like Me?": Underrepresented Groups in Science, Technology and Engineering (WISE, Bradford, UK, 2014).

[3] C. Hill, C. Corbett, and A. St. Rose, Why so Few? Women in Science, Technology, Engineering, and Mathematics (American Association of University Women, Washington, DC, 2010).

[4] H. Shen, Mind the gender gap, Nature (London) 495, 22 (2013).

[5] L. E. Kost, S. J. Pollock, and N. D. Finkelstein, Characterizing the gender gap in introductory physics, Phys. Rev. ST Phys. Educ. Res. 5, 010101 (2009).
[6] M. Lorenzo, C. H. Crouch, and E. Mazur, Reducing the gender gap in the physics classroom, Am. J. Phys. 74, 118 (2006).

[7] S. J. Pollock, N. D. Finkelstein, and L. E. Kost, Reducing the gender gap in the physics classroom: How sufficient is interactive engagement? Phys. Rev. ST Phys. Educ. Res. 3, 010107 (2007).

[8] A. Madsen, S. B. McKagan, and E. C. Sayre, Gender gap on concept inventories in physics: What is consistent, what is inconsistent, and what factors influence the gap? Phys. Rev. ST Phys. Educ. Res. 9, 020121 (2013).

[9] L. E. Kost-Smith, S. J. Pollock, and N. D. Finkelstein, Gender disparities in second-semester college physics: The incremental effects of a smog of bias, Phys. Rev. ST Phys. Educ. Res. 6, 020112 (2010).

[10] A. Miyake, L. E. Kost-Smith, N. D. Finkelstein, S. J. Pollock, G. L. Cohen, and T. A. Ito, Reducing the gender 
achievement gap in college science: A classroom study of values affirmation, Science 330, 1234 (2010).

[11] L. E. Kost-Smith, S. J. Pollock, N. D. Finkelstein, G. L. Cohen, T. A. Ito, and A. Miyake, Replicating a selfaffirmation intervention to address gender differences: Successes and challenges, AIP Conf. Proc. 1413, 231 (2012).

[12] I. Rodriguez, E. Brewe, V. Sawtelle, and L. H. Kramer, Impact of equity models and statistical measures on interpretations of educational reform, Phys. Rev. ST Phys. Educ. Res. 8, 020103 (2012).

[13] S. Bates, R. Donnelly, C. MacPhee, D. Sands, M. Birch, and N. R. Walet, Gender differences in conceptual understanding of Newtonian mechanics: A UK cross-institution comparison, Eur. J. Phys. 34, 421 (2013).

[14] P. Felten, Principles of good practice in SoTL, Teaching \& Learning Inquiry: The ISSOTL Journal 1, 121 (2013).

[15] E. Mazur, Peer Instruction: A User's Manual (Prentice Hall, Upper Saddle River, NJ, 1997).

[16] P. Heller, R. Keith, and S. Anderson, Teaching problem solving through cooperative grouping. Part 1: Group versus individual problem solving, Am. J. Phys. 60, 627 (1992).

[17] P. Heller and M. Hollabaugh, Teaching problem solving through cooperative grouping. Part 2: Designing problems and structuring groups, Am. J. Phys. 60, 637 (1992).

[18] H. D. Young, R. A. Freedman, and A. Lewis Ford, Sears and Zemansky's University Physics: With Modern Physics, 13th ed. (Pearson, Addison Wesley, San Francisco, 2012).

[19] E. K. Henriksen, J. Dillon, and J. Ryder, Understanding Student Participation and Choice in Science and Technology Education (Springer, Netherlands, 2015).

[20] H. T. Holmegaard, L. M. Ulriksen, and L. M. Madsen, The process of choosing what to study: A longitudinal study of upper secondary students' identity work when choosing higher education, Scandinavian Journal of educational research 58, 21 (2014).

[21] E. T. Pascarella and P. T. Terenzini, How College Affects Students: A Third Decade of Research (Jossey-Bass, San Francisco, CA, 2005), Vol. 2.

[22] G. D. Kuh, J. Kinzie, J. H. Schuh, and E. J. Whitt, Student Success in College: Creating Conditions that Matter (John Wiley \& Sons, New York, 2011).

[23] V. Tinto, From theory to action: Exploring the institutional conditions for student retention, in Higher Education: Handbook of Theory and Research, edited by J. C. Smart (Springer, Netherlands, 2010), pp. 51-89.

[24] L. Ulriksen, L. M. Madsen, and H. T. Holmegaard, What do we know about explanations for drop out/opt out among young people from STM higher education programmes?, Stud. Sci. Educ. 46, 209 (2010).

[25] M. W. Jorgensen and L. J. Phillips, Discourse Analysis as Theory and Method (Sage, London, Thousand Oaks, CA, 2002).

[26] J. P. Gee, An Introduction to Discourse Analysis: Theory and Method, 3rd ed. (Routledge, New York, 2011).

[27] E. G. Guba and Y.S. Lincoln, Competing paradigms in qualitative research, in Handbook of Qualitative Research, edited by N. K. Denzin and Y. S. Lincoln (Sage, Thousand Oaks, CA, 1994), pp. 105-117.
[28] S. Harding, The Science Question in Feminism (Cornell University Press, Ithaca, NY, 1986).

[29] K. Crenshaw, Mapping the margins: Intersectionality, identity politics, and violence against women of color, Stanford Law Rev. 43, 1241 (1991).

[30] Z. Hazari, P. M. Sadler, and G. Sonnert, The science identity of college students: Exploring the intersection of gender, race, and ethnicity, J. Coll. Sci. Teach. 42, 82 (2013).

[31] S. Kvale and S. Brinkmann, InterViews: Learning the craft of qualitative research interviewing, 2nd ed. (Sage, Los Angeles, 2008).

[32] C. Robson, Real World Research: A Resource for Social Scientists and Practitioner-Researchers, 2nd ed. (Blackwell, Oxford, 2002).

[33] F. Marton and R. Säljö, Approaches to learning, in The Experience of Learning: Implications for Teaching and Studying in Higher Education, 2nd ed., edited by F. Marton, D. J. Hounsell, and N. J. Entwistle (Scottish Academic Press, Edinburgh, 1997), pp. 39-58.

[34] P. Ramsden, Learning to Teach in Higher Education, 2nd ed. (Routledge, Falmer, London, 2003).

[35] G. S. Aikenhead, Students' ease in crossing cultural borders into school science, Sci. Educ. 85, 180 (2001).

[36] G. S. Aikenhead, Science Education for Everyday Life: Evidence-Based Practice (Teachers College Press, New York, 2006).

[37] S. Sweden, Yearbook of Educational Statistics 2015, Official Statistics of Sweden (Statistics Sweden, Stockholm, 2014).

[38] J. Sikora and A. Pokropek, Gender segregation of adolescent science career plans in 50 countries, Sci. Educ. 96, 234 (2012).

[39] C. Schreiner, Ph.D. thesis, University of Oslo, Oslo, Norway, 2006.

[40] S. Traweek, Beamtimes and Lifetimes: The World of High Energy Physicists (Harvard University Press, Cambridge, MA, 1988).

[41] C. Hasse, A. B. Sinding, S. Trentemøller, K. Velbaum, E. Lõhkivi, M.-L. Tina, J. Vainio, A. M. Ajello, C. Belardi, and G. Calafiore, Draw the Line! Universitites as Workplaces for Male and Female Researchers in Europe (UPGEM, Understanding Puzzles in the Gendered European Map, Tartu, 2008).

[42] A. J. Gonsalves, 'Physics and the girly girl-there is a contradiction somewhere': Doctoral students' positioning around discourses of gender and competence in physics, Cult. Stud. Sci. Educ. 9, 503 (2014).

[43] R. Tytler, Attitudes, identity, and aspirations toward science, in Handbook of Research on Science Education, edited by N. G. Lederman and S. K. Abell (Routledge, New York, 2014), Vol. II, pp. 82-103.

[44] Department of Education and Professional Studies, ASPIRES: Young People's Science and Career Aspirations, Age 10-14 (Kings College, London, 2013).

[45] L. Archer, J. DeWitt, J. Osborne, J. Dillon, B. Willis, and B. Wong, 'Balancing acts': Elementary school girls' negotiations of femininity, achievement, and science, Sci. Educ. 96, 967 (2012). 
[46] S. Andersson and C. Linder, Relations between motives, academic achievement and retention in the first year of a master programme in engineering physics, in Contemporary Science Education Research: Learning and Assessment, edited by G. Çakmakci and M. Fatih Taşar (Pegem Akademi, Ankara, 2010), pp. 123-128.

[47] C. M. Steele, Whistling Vivaldi: How Stereotypes Affect Us and What We Can Do (Norton, New York, 2011).

[48] K. Scantlebury, Gender matters: Building on the past, recognizing the present, and looking toward the future, in
Handbook of Research on Science Education, edited by N. G. Lederman and S. K. Abell (Routledge, New York, 2014), Vol. II, pp. 187-203.

[49] A. Hussénius, K. Andersson, A. Gullberg, and K. Scantlebury, Ignoring half the sky: A feminist critique of science education's knowledge society, in Science Education for Diversity, edited by N. Mansour and R. Wegerif (Springer, Netherlands, 2013), pp. 301-315.

[50] E. F. Redish, Teaching Physics with the Physics Suite (Wiley, Hoboken, NJ, 2003). 